\title{
Reactive cholecystitis as the leading sign of subacute perforation of the right ventricle with the electrode of an implantable cardioverter defibrillator
}

\author{
JASNA ČERKEZ HABEK ${ }^{1}$, ŽELJKA BELOŠIC HALLE ${ }^{3}$, HRVOJE GAŠPAROVIĆ4, TOMIS- \\ ALV JURKOVIĆ ${ }^{5}$, NENAD LAKUŠIĆ ${ }^{6}$, JOZICA ŠIKIĆ ${ }^{2}$
}

\author{
1 Department of Cardiovascular Diseases, University Hospital „Sveti Duh“, Zagreb, Croatia \\ Croatian Catholic University. \\ 2 Department of Cardiovascular Diseases, University Hospital „Sveti Duh“, Zagreb, Croatia \\ School of Medicine, University of Zagreb. \\ 3 Department of Gastroentrology, University Hospital ,, Sveti Duh", Zagreb, Croatia \\ 4 Department of Cardiosurgery, University Hospital Zagreb, Croatia \\ School of Medicine, University of Zagreb. \\ 5 Department of Radiology, University Hospital „Sveti Duh“, Zagreb, Croatia \\ 6 Special Hospital for Rehabilitation, Krapinske Toplice, Croatia \\ School of Medicine, University Josip Juraj Strossmayer, Osijek
}

Corresponding author:

Jasna Čerkez Habek

Department of Internal Diseases, Cardiovascular Unit

University Hospital „Sveti Duh“, Zagreb

Sveti Duh 64, 10000 Zagreb, Croatia

Fax: 0038513745534

E-mail: jasna.habek@gmail.com

\begin{abstract}
Subacute lead perforation of the right ventricle caused acute, reactive, acalculous cholecystis, which initially distracted the attention of physicians from the development of hematopericard. Implantation of a cardioverter defibrillator in a young patient after sudden cardiac arrest, but before treatment of significant stenosis of the proximal left anterior descending artery, resulted in a life-threatening condition only 36 days after arrest. After removing the implantable cardioverter defibrillator, there was no sign of pathological cardiac rhythm disorders.
\end{abstract}

Key words: subacute lead perforation of the right ventricle, reactive acalculos cholecystitis

\section{INTRODUCTION}

Sudden cardiac death (SCD) is a sudden, unexpected death caused by loss of heart function, and is responsible for half of all heart disease deaths. SCD occurs most frequently in adults in their mid-30s to mid-40s, and affects men twice as often as it does women. (1) Cardiovascular diseases are responsible for approximately 17 million deaths every year in the world, approximately $25 \%$ of which are SCD. Despite all diagnostic possibilities, up to $50 \%$ of cardiac arrests occur in individuals without known heart disease. (2) Prevention of SCD is aimed at reduction of risk factors for coronary artery disease, optimal treatment of patients with acute coronary syndrome and with ischemic cardiomyopathy. Recent trials have demonstrated the benefit of implantable cardioverter defibrillators (ICD). On the other hand, ICD may cause complications; one of them is described in this case report.

\section{CASE PRESENTATION}

A 41-year-old Caucasian man presented to the emergency department with a 2-day history of pain under the right costal arch, nausea, vomiting, diarrhea, hyperhidrosis and syncope. One month earlier, according to his medical records, the patient had a cardiac arrest on the highway. The driver performed basic life support and called the emergency team, who defibrillated the patient with $200 \mathrm{~J}$ for ventricular fibrillation. The patient was then transferred to the nearest hospital, where therapeutic hypothermia was immediately initiated, and a work up was done to determine the cause of arrest. Pulmonary angiography with multi-slice computed tomography
(MSCT) excluded pulmonary embolism, while coronary angiography revealed a $50 \%$ stenosis of the left main coronary artery and 50\%-75\% stenosis of the proximal left anterior descending artery (LAD). As the patient had repetitive non-sustained ventricular tachycardias (nsVT), an implantable cardioverter defibrillator (ICD) was inserted on post-arrest day 18. The day after, coronary angiography was repeated and the fractional flow reserve method was performed. The proximal LAD lesion was evaluated as significant, thus a stent was implanted with optimal angiographic result. The patient was discharged from the hospital on post-arrest day 30 , in good general condition. He had felt well until 3 days before he presented to our emergency department, when he experienced sudden, sharp pain described as if "the stent was scratching him". On the day of admission, 36 days had elapsed from cardiac arrest. The patient was normotensive, tachycardiac, eupnoic, afebrile, with normal breath sounds. The abdomen was sensitive on palpation under the right costal arch, soft, with audible peristalsis. Laboratory findings: L 15.5x109/L; E 3.85x1012/L; Hb 124 g/L; D dimers $5166 \mu \mathrm{g} / \mathrm{L}$; AST $43 \mathrm{U} / \mathrm{L}$; ALT $79 \mathrm{U} / \mathrm{L}$; CRP $2.7 \mathrm{mg} / \mathrm{L}$; troponin I 0.0 $\mu \mathrm{g} / \mathrm{L}$.

Heart and lung x-ray: no signs of infiltration on lung lobes or of respiratory fail- 


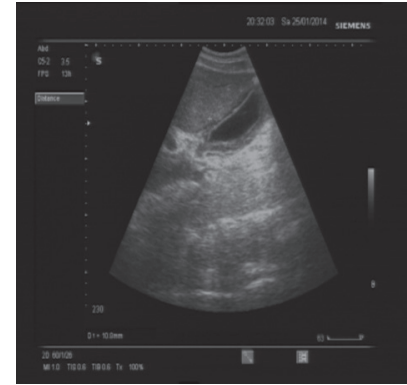

Figure 1. Acalculous cholecystitis.

ure; heart shadow enlarged, of hypertonic shape.

Electrocardiogram (ECG): sinus tachycardia, 114/min, intermedial axis, no ST/T changes.

Abdominal ultrasonography (US): a narrow trace of free liquid around the liver; thickened, inhomogeneous gallbladder walls, without pathologic contents within the gallbladder (figure 1) and minor pleural effusion on the left.

The patient was hospitalized under the diagnosis of acute acalculous cholecystitis. The next morning a repeat abdominal US still revealed signs of cholecystitis, with bilateral pleural and pericardial effusions. Echocardiography showed heart cavities of normal dimensions, hypertrophy of the interventricular septum, which was discretely hypokinetic, pericardial effusion at $8 \mathrm{~mm}$ before the right ventricle and 17 $\mathrm{mm}$ behind the posterior wall of the left ventricle and a left ventricle ejection fraction (EF LV) of $45 \%-50 \%$. The ICD electrode passed through the right atrium and right ventricle, with the tip of the electrode passing through the right ventricular wall and ending up in the pericardial effusion (figure 2).

The exact site of the electrode tip could not be visualized with certainty on MSCT of the thorax, due to reverberations of the metal part of the lead (figure 3).

The patient was transferred to the cardiac surgery department, where thoracotomy was performed and $200 \mathrm{~mL}$ of sanguinate pericardial effusion was removed after opening the pericardium. The right ventricle was perforated by the ICD lead, but there was no hemorrhage at the site of the
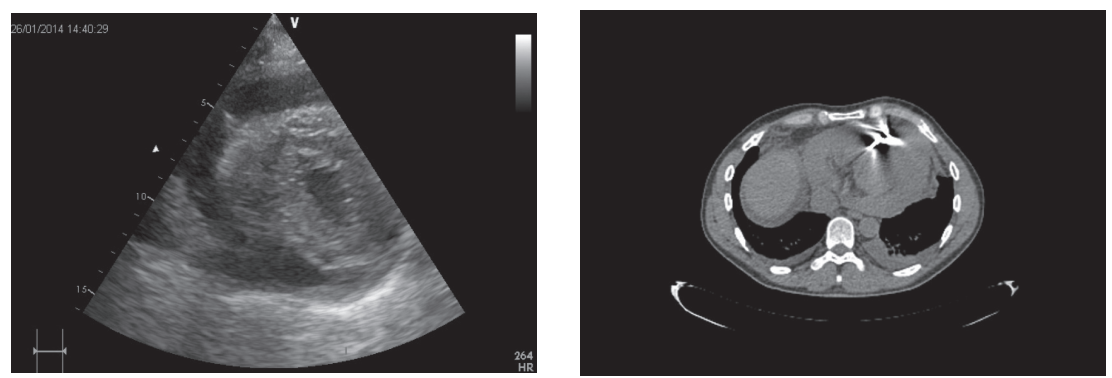

Figure 2. The intracardial defibrillator electrode seen outside the wall of the right ventricle.

rupture, with the lead in its position. A minor hematoma was found and evacuated. Upon extraction of the ICD lead, active hemorrhage was observed at the site of perforation, which was closed with two sutures. The ICD lead and the battery were removed. Before discharge from hospital, the patient underwent a 8.5 minutes exercise test at oxygen consumption of 34.65 $\mathrm{mL} / \mathrm{kg} / \mathrm{min}$, metabolic equivalents of task (METs) 9.9 and 79\% theoretical maximum; without malignant arrhythmias and clinical or ECG findings of coronary insufficiency.

During cardiac rehabilitation, the patient had no complaints. Noninvasive tests showed no malignant ventricular arrhythmia or coronary insufficiency.

\section{DISCUSSION}

Lead perforation is a rare complication of ICD, the incidence ranging from $0.6 \%$ to $5.2 \%$. (3) Ventricular perforations are divided into acute (within $24 \mathrm{~h}$ of placement), subacute (5-29 days after placement), and delayed (more than 30 days after placement). (4) Subacute and delayed perforations are rare and often not recognized. Acalculous cholecystitis occurs in $10 \%$ of all cases, usually associated with more serious morbidity and higher mortality rates, and traditionally observed in critically ill patients. (5) But, it has also been reported in outpatients without typical predisposing critical illness. (6) In spite of these reports, we believe that in the case of acute acalculous cholecystitis, physicians should always take into account the possibility of an underlying serious condition. Namely, the clinical condition of our patient was not seriously disturbed
Figure 3. Multislice computed tomography of the thorax: reverberation of the intracardial defibrillator lead, excluded the possibility of accurately determining its position.

and hence the diagnosis of "only" acute acalculous cholecystitis was made, which easily distracted the physician from more serious medical conditions. Appropriate management of the mechanical irritation and hematopericardium led to complete regression of the inflammatory lesions of the gallbladder. Diagnosis of both conditions was performed by ultrasound, while multi slice computer tomography was inferior because of reverberation of the foreign body. Subacute and/or late perforation of the right ventricle can be corrected by thoracotomy, in particular if the lead is in the mediastinum or other surrounding structures, with signs of hemorrhage; in some patients, it can be removed under fluoroscope or transesophageal US guidance, however, with a surgical team being ready to intervene. (7) In order to minimize the possibility of heart perforation, the lead should always be positioned along the septal wall or in the region of the right ventricle outflow tract. The most important point is that indication for all invasive procedures, as well as for ICD placement, should be carefully weighed. In the case presented, initial management of the significant proximal LAD stenosis followed by the decision for ICD implantation should have been optimal planning of therapeutic algorithm, especially considering the necessary balancing the benefits, possible complications and cost of a therapeutic procedure. After the ICD was removed, the patient had no malignant disorders of cardiac rhythm or signs of myocardial ischemia, which confirms our belief that after solving the critical LAD stenosis, implantation of an ICD was superfluous. Only 36 days after arrest, the patient's life was jeopardized again because of performing an "overprotective" procedure. 


\section{REFERENCES}

1. Mendis SPP, Norrving B. Global Atlas on Cardiovascular Disease Prevention and Control. Geneva: World Health Organization, 2011.

2. Eckart RE, Shry EA, Burke AP, McNear JA, Appel DA, Castillo-Rojas LM, Avedissian L, Pearse LA, Potter RN, Tremaine L, Gentlesk PJ, Huffer L, Reich SS, Stevenson WG. Department of Defense Cardiovascular Death Registry G. Sudden death in young adults: an autopsy-based series of a population undergoing active surveillance. J Am Coll Cardiol 2011;58:1254-61.

3. Welch AR, Yaday P, Lingle K. Subacute right ventricular pacemaker lead perforation: often talked about in consent forms but rarely seen. Cardiac Rhytm Management 2011;2:442-5.

4. Haq SA, Heitner JF, Lee L, Kassotis JT. Late presentation of a lead perforation as a complication of permanent pacemaker insertion. Angiology 2008;59:619-21.

5. McChesney J, Northump PG, Bickston SJ. Acute acalculose cholecystitis associated with systemic sepsis and visceral arterial hypoperfusion. Dig Dis Sci 2003;48:1960-7.

6. Ryu JK, Ryu KH, Kim KH. Clinical features of acute acalculous cholecystitis. J Clin Gastroenterol 2003;36:166-9.

7. Laborderie J, Barandon L, Ploux S. Management of subacute and delayed right ventricular perforation with a pacing or an implantable cardioverter-defibrillator lead. Am J Cardiol 2008;102:1352-5. 